\title{
Trapping of Single Atoms in Cavity QED
}

\author{
J. Ye,* D. W. Vernooy, and H. J. Kimble \\ Norman Bridge Laboratory of Physics, California Institute of Technology 12-33, Pasadena, California 91125
}

(Received 2 August 1999)

\begin{abstract}
By integrating the techniques of laser cooling and trapping with those of cavity quantum electrodynamics (QED), single cesium atoms have been trapped within the mode of a small, high finesse optical cavity in a regime of strong coupling. The observed lifetime for individual atoms trapped within the cavity mode is $\tau \approx 28 \mathrm{~ms}$, and is limited by fluctuations of light forces arising from the far-detuned intracavity field. This initial realization of trapped atoms in cavity QED should enable diverse protocols in quantum information science.
\end{abstract}

PACS numbers: 42.50.Vk, 03.67.-a, 32.80.Pj

Cavity quantum electrodynamics (QED) offers powerful possibilities for the deterministic control of atom-photon interactions quantum by quantum [1,2]. Indeed, modern experiments in cavity QED have achieved the exceptional circumstance of strong coupling, for which single quanta can profoundly impact the dynamics of the atom-cavity system. Cavity QED has led to many new phenomena, including the realization of a quantum phase gate [3], the creation of Fock states of the radiation field [4], and the demonstration of quantum nondemolition detection for single photons [5].

These and other diverse accomplishments set the stage for advances into yet broader frontiers in quantum information science for which cavity QED offers unique advantages. For example, it should be possible to realize complex quantum circuits and quantum networks by way of multiple atom-cavity systems linked by optical interconnects [6,7], as well as to pursue more general investigations of quantum dynamics for continuously observed open quantum systems [8]. The primary technical challenge on the road toward these scientific goals is the need to trap and localize atoms within a cavity in a setting suitable for strong coupling, thereby eliminating the indeterminism intrinsic to atom beams. In fact, all serious schemes for quantum computation and communication via cavity QED rely on developing techniques for atom confinement that do not interfere with cavity QED interactions.

In this Letter, we report a significant milestone in this quest, namely the first trapping of a single atom in cavity QED. Our experiment integrates the techniques of laser cooling and trapping with those of cavity QED to deliver cold atoms (kinetic energy $E_{k} \simeq 30 \mu \mathrm{K}$ ) into the mode of a high finesse optical cavity. In a domain of strong coupling, the trajectory of an individual atom within the cavity mode can be monitored in real time by a near resonant field with mean intracavity photon number $\bar{n}<$ 1 [9-13]. Here we exploit this capability to trigger $\mathrm{ON}$ an auxiliary field that functions as a far-off-resonance dipoleforce trap (FORT) [14,15], providing a confining potential to trap the atom within the cavity mode. Likewise, when the FORT is turned OFF after a variable delay, strong coupling enables detection of the atom. Repetition of such measurements yields a trap lifetime $\tau=28 \pm 6 \mathrm{~ms}$, which is currently limited by fluctuations in the intensity of the intracavity trapping field (FORT). Stated in units of the coupling parameter $g_{0}$ (where $2 g_{0}$ is the singlephoton Rabi frequency), our work achieves $g_{0} \tau \simeq 10^{6} \pi$, whereas prior experiments with cold atoms have attained $g_{0} T \simeq 10^{4} \pi$ [9-13] and experiments with conventional atomic beams have $g_{0} T \simeq \pi[1-5]$, with $T$ as the atomic transit time through the cavity mode.

Our apparatus is depicted in Fig. 1. Roughly $10^{8}$ cesium atoms are accumulated in an "upstairs" MOT-1, cooled with polarization gradients to $3 \mu \mathrm{K}$, and then transferred with $10 \%$ efficiency to a "downstairs" MOT-2 located in a UHV chamber with background pressure $10^{-10}$ Torr [Fig. 1(c)]. The atoms captured in MOT-2 are next cooled to $2 \mu \mathrm{K}$ and dropped from a position $5 \mathrm{~mm}$ above an optical cavity. A final stage to deliver cold atoms into the cavity mode is provided by a set of cooling beams located in the $y-z$ plane perpendicular to the horizontal cavity axis [Fig. 1(b)]. These beams form two independent standing waves along the $\pm 45^{\circ}$ directions in the $y-z$ plane, each with helical polarization, and are switched to remove the residual fall velocity of atoms arriving at the cavity mode from MOT-2, leading to final velocities $v \sim 5 \mathrm{~cm} / \mathrm{s}$ for atoms in the immediate vicinity of the cavity mode.

The Fabry-Pérot cavity into which the atoms fall is formed from two superpolished spherical mirrors. The cavity length $l=44.6 \mu \mathrm{m}$, waist $w_{0}=20 \mu \mathrm{m}$, and finesse $\mathcal{F}=4.2 \times 10^{5}$, and hence a cavity field decay rate $\kappa / 2 \pi=4 \mathrm{MHz}$ [16]. The atomic transition employed for cavity QED is the $\left(g \equiv 6 S_{1 / 2}, F=4, m_{F}=4 \rightarrow\right.$ $\left.e \equiv 6 P_{3 / 2}, F=5, m_{F}=5\right)$ component of the $D_{2}$ line of atomic cesium at $\lambda_{\text {atom }} \equiv c / \nu_{\text {atom }}=852.4 \mathrm{~nm}$. Here, $\quad\left(g_{0}, \gamma_{\perp}\right) / 2 \pi=(32,2.6) \mathrm{MHz}$, with $g_{0}$ as the peak atom-field coupling coefficient and $\gamma_{\perp}$ as the dipole decay rate for the $e \rightarrow g$ transition, leading to critical photon and atom numbers $\left(m_{0} \equiv \gamma_{\perp}^{2} / 2 g_{0}^{2}, N_{0} \equiv 2 \kappa \gamma_{\perp} / g_{0}^{2}\right)=(0.003,0.02)$.

The cavity length is stabilized with an auxiliary diode laser $\lambda_{\text {lock }} \equiv c / \nu_{\text {lock }} \approx 836 \mathrm{~nm}$, which is itself stabilized 


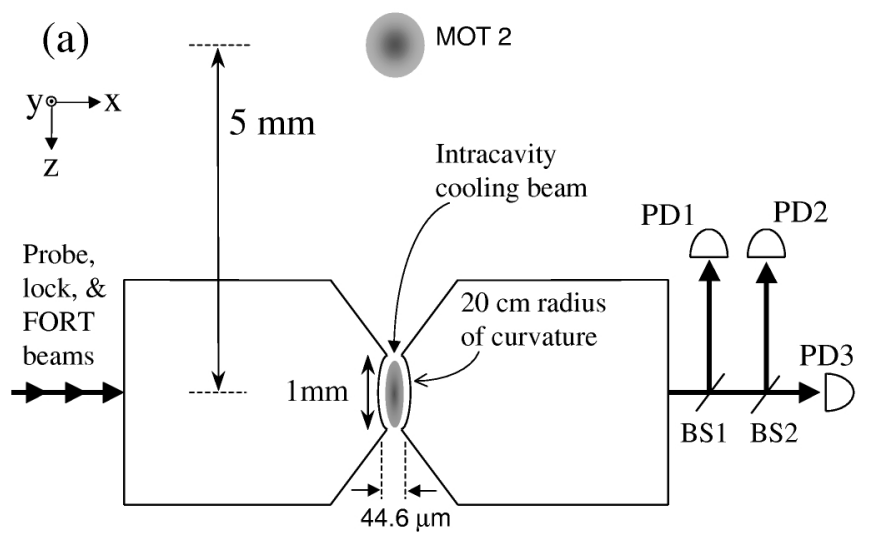

(b)

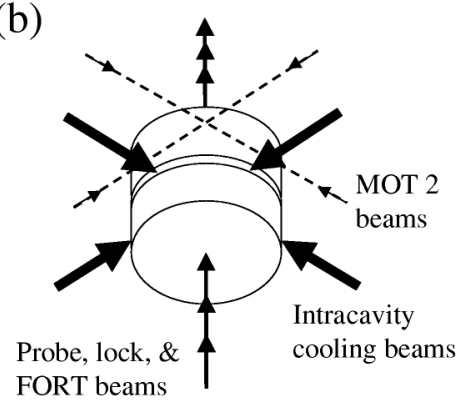

(c)

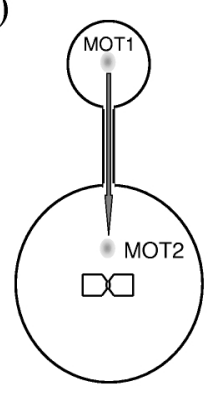

FIG. 1. Schematic of the experimental apparatus. (a) The dichroic beam splitter BS1 sends the cavity-length-stabilizing beam to PD1. BS2 separates the FORT (sent to PD2 for locking) and cavity QED beams (sent to PD3 for balanced heterodyne detection). (b) Beam geometry for intracavity cooling and MOT-2. (c) Differentially pumped chamber and the two-stage MOT.

relative to $\nu_{\text {atom }}$ by way of an auxiliary "transfer cavity" [11,17]. It is detuned 2 longitudinal-mode orders above the cavity QED mode at $\nu_{\text {cavity }} \approx \nu_{\text {atom }}$, and creates a small ac Stark shift of $50 \mathrm{kHz}$ in $\nu_{\text {atom. Residual length }}$ fluctuations lead to variations in $\Delta_{\text {ac }} \equiv \nu_{\text {atom }}-\nu_{\text {cavity }}$ of $\delta \Delta_{\mathrm{ac}} \approx \pm 10 \mathrm{kHz}$ contained within a locking bandwidth of about $10 \mathrm{kHz}$.

The "trajectory" of an individual atom is monitored in real time as it enters and moves within the cavity mode by recording large fractional modifications of the ( $\mathrm{pW}$ scale) cavity transmission for a circularly polarized probe field $\mathcal{E}_{\text {probe }}$ of frequency $\nu_{\text {probe }}=$ $\nu_{\text {atom }}+\Delta_{\text {probe }}$. The spatially dependent coupling coefficient $g(\vec{r})=g_{0} \sin \left(2 \pi x / \lambda_{\text {cavity }}\right) \exp \left[-\left(y^{2}+z^{2}\right) / w_{0}^{2}\right] \equiv$ $g_{0} \psi\left(\vec{r}, \lambda_{\text {cavity }}\right)$, with the mirrors located at $x=(0, l)$. Heterodyne detection of the transmitted field (with overall efficiency 47\%) allows inference of the atomic position in a fashion that can be close to the standard quantum limit [11].

For the purpose of atomic trapping, the transmitted probe beam can be employed to trigger ON a far-offresonance trap (FORT) [14,15] given the detection of an atom entering the mode volume. Here, the FORT beam is derived from an external diode laser locked to a cavity mode at $\lambda_{\mathrm{FORT}}=c / \nu_{\mathrm{FORT}}=869 \mathrm{~nm}$, two longitudinalmode orders below the cavity QED mode at $\nu_{\text {cavity. In }}$ this case, the standing-wave patterns of the two modes at $\left(\nu_{\text {FORT }}, \nu_{\text {cavity }}\right)$ have approximately coincident antinodes near the center and ends of the cavity.

An example of the trapping of a single atom is given in Fig. 2. In (a) the arrival of an atom is sensed by a reduction in transmission of $\mathcal{E}_{\text {probe }}$ (with intracavity photon number $\bar{n} \approx 0.1$ [18]). The falling edge of the probe transmission triggers ON the FORT field, which then remains on until being switched OFF after a fixed interval. The presence of the atom at this OFF time is likewise detected by modification of the probe transmission, demonstrating a trapping time of $13.5 \mathrm{~ms}$ for the particular event shown. Note that because the conditional probabilities for atom trapping given a trigger $p_{\text {tp } \mid \mathrm{tg}}$ and for detection given a trapped atom $p_{\mathrm{d} \mid \mathrm{tp}}$ are rather small $\left(p_{\mathrm{tp} \mid \mathrm{tg}} p_{\mathrm{d} \mid \mathrm{tp}} \sim 0.03\right)$, we operate at rather high densities of cold atoms, such that the average intracavity atom number at the time of the trigger is $\bar{N}_{\text {atom }} \sim 0.5$ (but which then falls off rapidly). As a
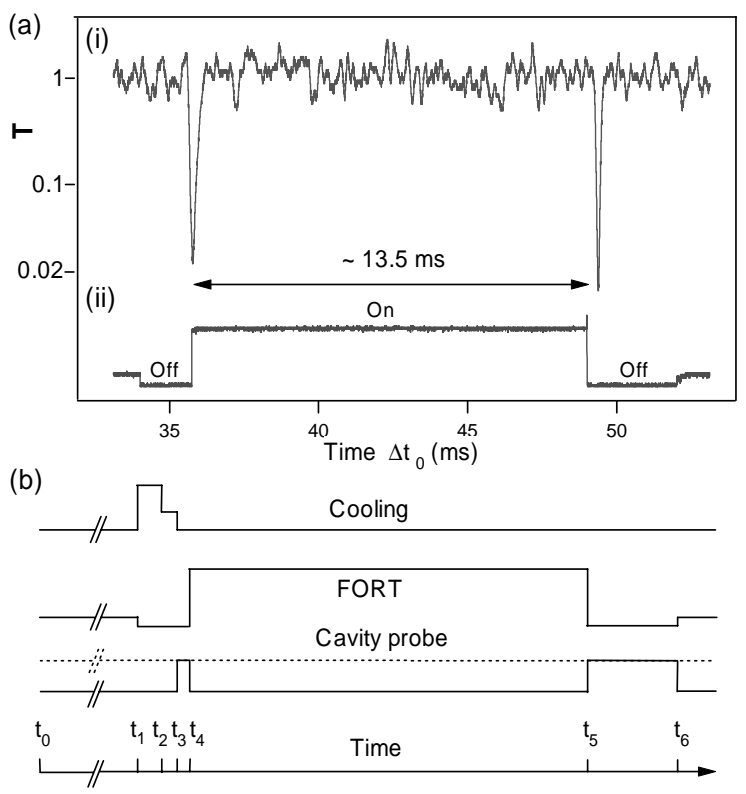

FIG. 2. (a) A single atom [curve (i), with $30 \mathrm{kHz}$ bandwidth] is detected within the cavity mode and triggers ON a dipoleforce trap [FORT, curve (ii)]. When the FORT is switched OFF after a $13.5 \mathrm{~ms}$ delay, the atom is detected again. $\Delta_{\text {probe }}=$ $0=\Delta_{a c}, \Delta_{\mathrm{FORT}}^{g}=-45 \mathrm{MHz}$, and $\bar{n}=0.1$ photons. Note that $T$ is normalized to the transmission for the empty cavity (no atom). (b) Timing sequence. The polarization-gradient cooled atoms from MOT-2 are released at time $t_{0}(0 \mathrm{~ms})$. With no intracavity cooling [Fig. 1(b)] and no FORT, atoms reach the cavity mode between 27 to $37 \mathrm{~ms}$. The FORT is switched $\mathrm{OFF}$ and the cooling beams are switched $\mathrm{ON}$ at $t_{1}(34 \mathrm{~ms})$, with then the cooling beams detuned further by $18 \mathrm{MHz}$ and their intensities decreased between $t_{2}(35 \mathrm{~ms})$ and $t_{3}(35.5 \mathrm{~ms})$. At $t_{3}$ the cavity probe is turned ON. Atomic detection, for example at $t_{4}$, triggers the FORT $\mathrm{ON}$ and the cavity probe OFF. After a predetermined delay $\left(t_{5}-t_{4}\right)$ the FORT is turned OFF and the cavity probe ON for detection $\left(t_{5}\right)$, with then everything reset at $t_{6}$. For trace (a)(i), the cavity probe was left on continuously [dashed line in (b)]. 
consequence, the atom that causes the trigger is not always the atom that is actually trapped when the FORT is gated ON, with such "phantom" events estimated to occur in roughly 1 of 4 cases.

The timing diagram for switching of the various fields is given in Fig. 2(b). Note that although the probe field is left on for all times in Fig. 2(a), there is no apparent change in cavity transmission during the interval in which an atom is purportedly trapped within the cavity mode. The absence of atomic signatures during the trapping time, but not before or after, is due to ac Stark shifts associated with the FORT and/or the mismatched antinodes between $\left(\nu_{\mathrm{FORT}}, \nu_{\text {cavity }}\right)$. For the data of Fig. 2 , a power of $30 \mu \mathrm{W}$ incident upon the cavity at $\lambda_{\mathrm{FORT}}$ leads to a circulating intracavity power of $1 \mathrm{~W}$, and to ac Stark shifts $\Delta_{\mathrm{FORT}}^{e, g}=$ $\pm 45 \mathrm{MHz}$ for $e, g$ at the cavity antinodes, so that at these locations, the net atomic transition frequency $\nu_{\text {atom }}$ is blueshifted by $\Delta_{\mathrm{FORT}}^{e}-\Delta_{\mathrm{FORT}}^{g} \equiv \Delta_{\mathrm{FORT}}=+90 \mathrm{MHz}$. Moreover, the spatial dependence of the cavity mode means that $\Delta_{\mathrm{FORT}}^{e, g}(\vec{r})=\Delta_{\mathrm{FORT}}^{e, g} \psi\left(\vec{r}, \lambda_{\mathrm{FORT}}\right)$, so that the FORT effectively provides a spatially dependent detuning that shifts the cavity QED interactions out of resonance, with $\Delta_{\mathrm{ac}} \rightarrow \Delta_{\mathrm{ac}}+\Delta_{\mathrm{FORT}}(\vec{r})$. The probe transmission in this case requires an analysis of the eigenvalue structure incorporating both $g_{0}(\vec{r})$ as well as $\Delta_{\mathrm{FORT}}(\vec{r})$ [19].

To avoid questions related to the complexity of this eigenvalue structure as well as to possible heating or cooling by the probe field, we synchronously gate OFF the probe field $\mathcal{E}_{\text {probe }}$ for measurements of trap lifetime, with one such result displayed in Fig. 3. These data are acquired for repeated trials as in Fig. 2 (namely, with the presence of an atom used to trigger $\mathrm{ON}$ the FORT of depth $\Delta_{\text {FORT }}^{g}=-50 \mathrm{MHz}$ ), but now with the probe field $\mathcal{E}_{\text {probe }}$ gated OFF after receipt of a valid trigger. At the end of the trapping interval, $\mathcal{E}_{\text {probe }}$ is gated back ON, and the success (or failure) of atomic detection recorded. The lifetime for single atoms trapped within the FORT is thereby determined to be $\tau_{\mathrm{FORT}}=$

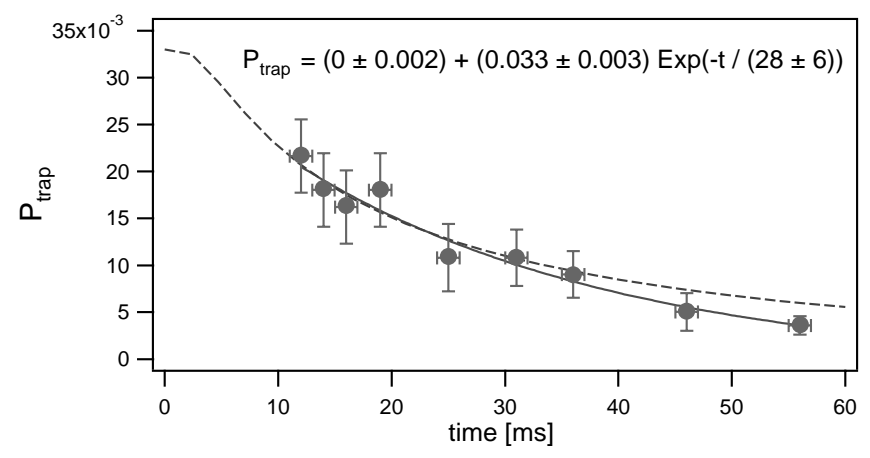

FIG. 3. Measurement of trap lifetime $\tau_{\text {FORT }}$ (see text) with the FORT triggered $\mathrm{ON}$ by single atom transits [e.g., $t_{4}$ of Fig. 2(b)]. $P_{\text {trap }}$ gives the probability per trigger of successful detection of a trapped atom in the time window $\left(t_{5}, t_{6}\right)$. The exponential fit results in $\tau_{\mathrm{FORT}}=28 \pm 6 \mathrm{~ms}$. The dashed curve is from the theory of Ref. [21].
$(28 \pm 6) \mathrm{ms}$. This trap lifetime is confirmed in an independent experiment where the FORT is turned on and off at predetermined times without transit triggering (with reduced trapping probability but with otherwise similar operation procedures), yielding $\tau_{\mathrm{FORT}}^{\prime}=(27 \pm 6) \mathrm{ms}$. As mentioned in the discussion of Fig. 2, our ability to load the trap with reasonable efficiency via asynchronous turn on is due to operation with large $\bar{N}_{\text {atom }}$.

Note that at each of the time delays in Fig. 3, a subtraction of "background" events (atomic transits delayed by the intracavity cooling beams) has been made from the set of total detected events. We determine this background by way of measurements following the same protocol as in Fig. 2(b), but without the FORT beam. For times below $10 \mathrm{~ms}$ in Fig. 3, this background dominates the signal by roughly 50 -fold, precluding accurate measurements of trapped events. However, because it has a rapid decay time of $\approx 3 \mathrm{~ms}$, for times greater than about $20 \mathrm{~ms}$ it makes a negligible contribution.

As for the factors that limit the trap lifetime, the spontaneous photon scattering rate is $37 \mathrm{~s}^{-1}$ in our FORT (recall that the cesium recoil frequency is $\sim 2 \mathrm{kHz}$ and the trap depth $\sim 50 \mathrm{MHz}$ ). The trap lifetime set by background gas collisions at a pressure of $10^{-10}$ Torr is estimated to be $\sim 100 \mathrm{~s}$, which is likewise much longer than that actually observed. However, Savard et al. [20] have shown that laser intensity noise causes heating in a FORT with heating rate $\tau_{e}^{-1}=\pi^{2} \nu_{\text {tr }}^{2} S_{e}\left(2 \nu_{\text {tr }}\right)$ [Eq. (12) of Ref. [20]]. Here $\nu_{\text {tr }}$ is the trap oscillation frequency (in cycles/s) and $S_{e}\left(2 \nu_{\mathrm{tr}}\right)$ is the power spectral density of fractional intensity noise evaluated at frequency $2 \nu_{t r}$. For the FORT of Fig. 3, we estimate $\left(\nu_{\mathrm{tr}}^{\text {radial }}, \nu_{\mathrm{tr}}^{\text {axial }}\right) \approx(5,450) \mathrm{kHz}$ for the radial $(y, z)$ and axial $x$ directions, respectively. Direct measurements of the FORT beam emerging from the cavity lead to $\left[S_{e}\left(2 \nu_{\mathrm{tr}}^{\text {radial }}\right), S_{e}\left(2 \nu_{\mathrm{tr}}^{\text {axial }}\right)\right] \approx\left(5 \times 10^{-9}, 2.3 \times\right.$ $\left.10^{-11}\right) / \mathrm{Hz}$, so that $\left(\tau_{e}^{\text {radial }}, \tau_{e}^{\text {axial }}\right) \approx(830,23) \mathrm{ms}$. Given the heating rate of $1 / \tau_{e}^{\text {axial }}$, a solution of the stochastic master equation based upon the model of Ref. [20] provides a good description of the measured trap decay [21], as shown by the dashed curve in Fig. 3, leading to the conclusion that fluctuations in intracavity intensity drive heating along the cavity axis and are the limiting factor in this work.

Finally, we return to the more general question of cavity QED in the presence of the FORT. As a starting point in a more complete investigation, Fig. 4 displays a series of four atomic transits, each of increasing duration. With the FORT OFF, the "down-going" transit in (a) arises from an atom that was dropped from MOT-2 without the application of the cooling pulse shown in Fig. 2(b) and provides a reference for the time of free fall through $\psi\left(\vec{r}, \lambda_{\text {cavity }}\right)$ (here, $T \approx 100 \mu$ s for $v \approx 30 \mathrm{~cm} / \mathrm{s}$ ). By contrast, with the cooling pulse applied (but with the FORT still OFF), the transit in (b) is lengthened to $T \approx$ $420 \mu \mathrm{s}$. In (c), $\Delta_{\text {probe }}$ is altered to sense "up-going" transits [10], with now $T \approx 1 \mathrm{~ms}$. Because the kinetic 

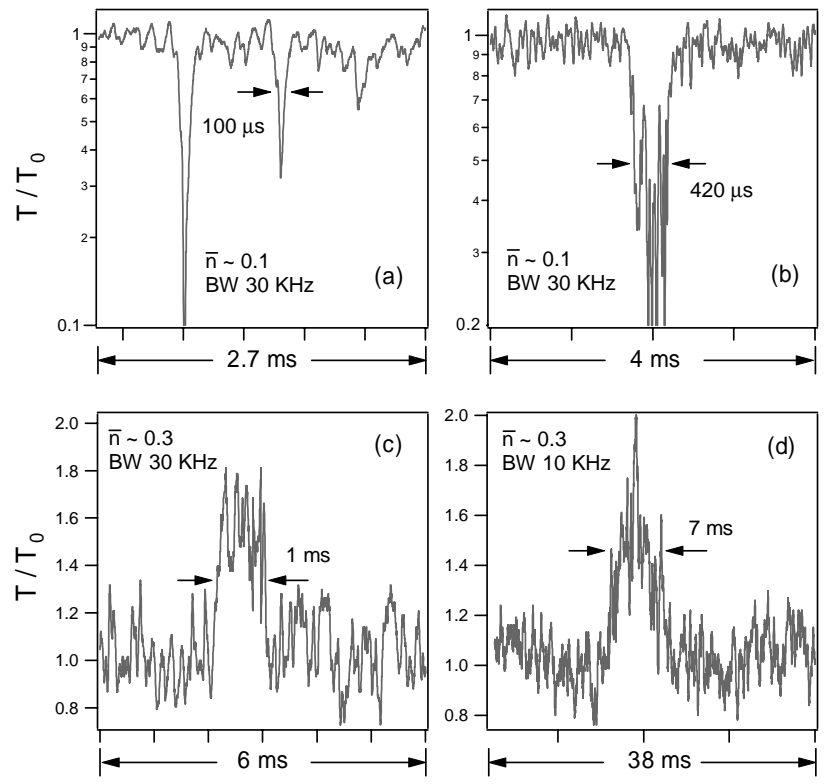

FIG. 4. Representative transits under four different conditions. (a) Atom free falls, $\Delta_{\text {probe }}=0=\Delta_{\text {ac }}$. (b) Intracavity cooling $\mathrm{ON}, \Delta_{\text {probe }}=0=\Delta_{\mathrm{ac}}$. (c) Intracavity cooling $\mathrm{ON}$, $\Delta_{\text {probe }}=-30 \mathrm{MHz}, \Delta_{\mathrm{ac}}=0$. (d) Both intracavity cooling and FORT ON, $\Delta_{\text {probe }}=-10 \mathrm{MHz}, \Delta_{\mathrm{ac}}=-10 \mathrm{MHz}$, and $\Delta_{\text {FORT }}^{g}=-15 \mathrm{MHz}$.

energy of an atom with $v \sim 5 \mathrm{~cm} / \mathrm{s}$ is much smaller than the coherent coupling energy $\hbar g_{0}$, it is possible to achieve long localization times via the single-photon trapping and cooling mechanisms discussed in Refs. [22,23]. For the last trace in (d), the FORT is always ON (i.e., not gated as in Fig. 2), but with a shallower potential $\left(\Delta_{\text {FORT }}^{g}=\right.$ $-15 \mathrm{MHz})$ than that in Fig. 2(a). We select $\left(\Delta_{\text {probe }}=\right.$ $-10 \mathrm{MHz}, \Delta_{\mathrm{ac}}=-10 \mathrm{MHz}$ ) to enhance observation of a trapped atom via the composite eigenvalue structure associated with $g(\vec{r})$ and $\Delta_{\mathrm{FORT}}(\vec{r})$. We also expect that cavity-assisted Sisyphus cooling [23] should be effective in this setting. As in (d), this results in remarkable "transits" observed in real time with $T \approx 7 \mathrm{~ms}$, corresponding to a mean transit velocity $\bar{v} \equiv \frac{2 w_{0}}{T} \approx 6 \mathrm{~mm} / \mathrm{s}$.

In conclusion, although these are encouraging first results for trapping of single atoms in cavity QED, an outstanding problem with dipole-force traps is that the excited state experiences a positive ac Stark shift, leading to an excited state atom being repelled from the trap (e.g., during quantum logic operations). As well, the effective detuning $\Delta_{\mathrm{ac}}(\vec{r}) \equiv \Delta_{\mathrm{ac}}+\Delta_{\mathrm{FORT}}(\vec{r})$ is a strong function of the atom's position within the trap. Fortunately, it turns out that a judicious choice of $\lambda_{\text {FORT }}$ can eliminate both of these problems by making $\Delta_{\mathrm{FORT}}^{e}(\vec{r})=\Delta_{\mathrm{FORT}}^{g}(\vec{r})<0$, and hence $\Delta_{\text {FORT }}(\vec{r})=0$ [24]. Alternatively, even for the current setup, it is possible to tune $\Delta_{\mathrm{FORT}}^{e}$ together with $\Delta_{\mathrm{ac}}$ to produce regions within the cavity mode for which the spatially dependent level shift of a composite dressed state in the first excited manifold matches $\Delta_{\text {FORT }}^{g}(\vec{r})$ for the (trapping) ground state, as was attempted in
Fig. 4(d). These schemes in concert with extensions of the capabilities presented in this Letter should allow us to achieve atomic confinement in the Lamb-Dicke regime (i.e., $\left.\eta_{x} \equiv 2 \pi \Delta x / \lambda \ll 1\right)$ in a setting for which the trapping potential for the atomic center-of-mass motion is independent of internal atomic state, as has been so powerfully exploited with trapped ions [25]. Generally speaking, this essential task must be completed for longterm progress in quantum information science via photonatom interactions.

We enthusiastically acknowledge the contributions of C. J. Hood, T. W. Lynn, and H. Mabuchi. This work was funded by the NSF, by DARPA via the QUIC (Quantum Information and Computing) program administered by ARO, by the ONR, and by HP Research Labs.

*Present address: JILA, NIST, and University of Colorado, Boulder, CO.

[1] Cavity Quantum Electrodynamics, edited by P. Berman (Academic Press, San Diego, 1994).

[2] Special Issue on Modern Studies of Basic Quantum Concepts and Phenomena [Phys. Scr. T76 (1998)].

[3] Q. A. Turchette et al., Phys. Rev. Lett. 75, 4710 (1995).

[4] H. Walther et al., in Proceedings of the Fourteenth International Conference on Laser Spectroscopy (ICOLS99), Innsbruck, 1999, edited by R. Blatt, J. Eschner, D. Leibfried, and F. Schmidt-Kaler (to be published).

[5] G. Nogues et al., Nature (London) 400, 239 (1999).

[6] T. Pellizzari et al., Phys. Rev. Lett. 75, 3788 (1995).

[7] J.-I. Cirac et al., Phys. Scr. T76, 223 (1998).

[8] H. Mabuchi and H. Wiseman, Phys. Rev. Lett. 82, 1798 (1999).

[9] H. Mabuchi et al., Opt. Lett. 21, 1393 (1996).

[10] C. J. Hood et al., Phys. Rev. Lett. 80, 4157 (1998).

[11] H. Mabuchi, J. Ye, and H. J. Kimble, Appl. Phys. B 68, 1095 (1999).

[12] J. Ye et al., IEEE Trans. Instrum. Meas. 48, 608 (1999).

[13] P. Müstermann et al., Phys. Rev. Lett. 82, 3791 (1999).

[14] J. D. Miller, R. A. Cline, and D. J. Heinzen, Phys. Rev. A 47, R4567 (1993).

[15] H. J. Lee et al., Phys. Rev. Lett. 76, 2658 (1996).

[16] The differential phase shift between the cavity eigenpolarizations is $\Delta \beta=2 \mu \mathrm{rad} /$ bounce.

[17] J. Helmcke, S. A. Lee, and J. L. Hall, Appl. Opt. 21, 1686 (1982).

[18] Throughout, $\bar{n}$ specifies the mean intracavity photon number for the empty cavity (i.e., no atoms) at the actual detuning $\Delta_{\text {probe }}$.

[19] D. W. Vernooy and H. J. Kimble, Phys. Rev. A 56, 4287 (1997).

[20] T. A. Savard, K. M. O'Hara, and J. E. Thomas, Phys. Rev. A 56, R1095 (1997).

[21] C. W. Gardiner et al., quant-ph/9910056.

[22] A. C. Doherty et al., Phys. Rev. A 56, 833 (1997).

[23] P. Horak et al., Phys. Rev. Lett. 79, 4974 (1997).

[24] H. J. Kimble et al., in Proceedings of the Fourteenth International Conference on Laser Spectroscopy (Ref. [4]).

[25] D. Leibfried et al., Phys. Rev. Lett. 77, 4281 (1996). 